\title{
PSEUdoACALASIA: UM DESAFIO DIAGNÓSTICO
}

\section{Pseudoachalasia: a diagnostic challenge}

\author{
Gabriela Jungblut Schuh"1, João Edson Prediger ${ }^{1}$, Sandra Jungblut \\ Schuh $^{2}$, Rodrigo Soares de Souza Marques ${ }^{3}$, Antônio Carlos Maciel ${ }^{4}$
}

Clin Biomed Res. 2021;41(1):92-94

1 Faculdade de Medicina, Universidade Federal do Rio Grande do Sul (UFRGS). Porto Alegre, RS, Brasil.

2 Hospital Sanatório Partenon, Secretaria Estadual da Saúde do Estado do Rio Grande do Sul. Porto Alegre, RS, Brasil.

3 Departamento de Radiologia e Diagnóstico por Imagem, Hospital Santa Casa de Misericórdia de Porto Alegre. Porto Alegre, RS, Brasil.

4 Departamento de Radiologia e Diagnóstico por Imagem, Hospital de Clínicas de Porto Alegre. Porto Alegre, RS, Brasil.

Autor correspondente: Gabriela Jungblut Schuh gschuh@hcpa.edu.br Faculdade de Medicina, Universidade Federal do Rio Grande do Sul (UFRGS)

Rua Ramiro Barcelos, 2400. 90035-002, Porto Alegre, RS, Brasil.

\section{RESUMO}

Pseudoacalasia se manifesta de forma semelhante à acalasia do esfíncter esofágico inferior (EEI). Corresponde a 2,4 a $4 \%$ dos casos que simulam acalasia ${ }^{1}$. Estima-se que cerca de $50 \%$ dos casos de pseudoacalasia correspondam a malignidade primária do esôfago e da junção gastroesofágica e 18\% a malignidades secundárias como doença metastática ${ }^{1,2}$. Etiologias benignas também estão descritas. No presente trabalho apresentamos um caso de pseudoacalasia com ênfase nos aspectos radiológicos. Revisamos também aspectos clínicos e radiológicos que podem auxiliar no diagnóstico diferencial entre acalasia e pseudoacalasia.

Palavras-chave: Pseudoacalasia; neoplasias esofágicas; adenocarcinoma; disfagia; acalasia

\section{ABSTRACT}

Pseudoachalasia is a condition that mimics idiopathic achalasia of the lower esophageal sphincter. It accounts for 2.4 to $4 \%$ of patients presenting with achalasia-like symptoms ${ }^{1}$. It is estimated that about $50 \%$ of cases of pseudoachalasia correspond to primary malignancies of the esophagus and the esophagogastric junction and $18 \%$ to secondary malignancies such as metastase ${ }^{1,2}$. Benign causes are also described. In this report we emphasize radiological findings of a case of pseudoachalasia. We also review clinical and radiological aspects that might be auxiliary in the differential diagnosis between achalasia and pseudoachalasia.

Keywords: Pseudoachalasia; esophageal neoplasms; adenocarcinoma; dysphagia; achalasia

\section{CASO CLÍNICO}

Apresentamos imagens da radiografia contrastada e tomografia computadorizada de abdome de um paciente masculino, 57 anos, que apresentava disfagia, inicialmente para sólidos e posteriormente para líquidos, com piora progressiva em 7 meses e perda ponderal de $13 \mathrm{~kg}$. Realizou duas endoscopias que demonstraram o afilamento da cárdia sem lesão mucosa. Rigidez da cárdia impediu a passagem do endoscópio. Realizou radiografia contrastada do esôfago (Figura 1) e após tomografia computadorizada (Figuras 2 e 3). O diagnóstico anatomopatológico foi de adenocarcinoma da junção esofagogástrica. 


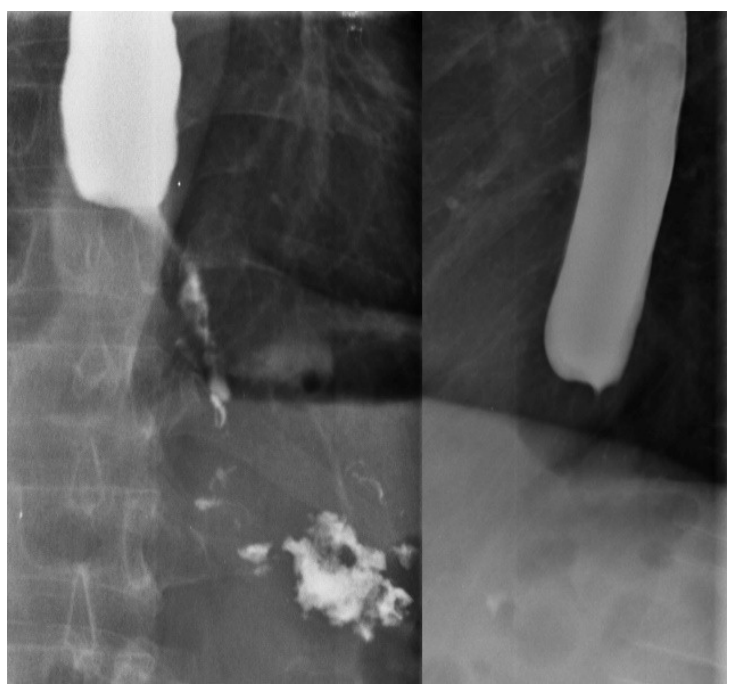

Figura 1: Radiografia contrastada com afilamento cônico, simétrico e com relevo mucoso liso da porção distal do esôfago, determinando estase do meio de contraste. A zona de afilamento é longa, com mais de $3,5 \mathrm{~cm}$. A restrição ao fluxo do meio de contraste limita avaliação da extremidade distal. O diâmetro do esôfago tubular foi de aproximadamente $3,5 \mathrm{~cm}$.

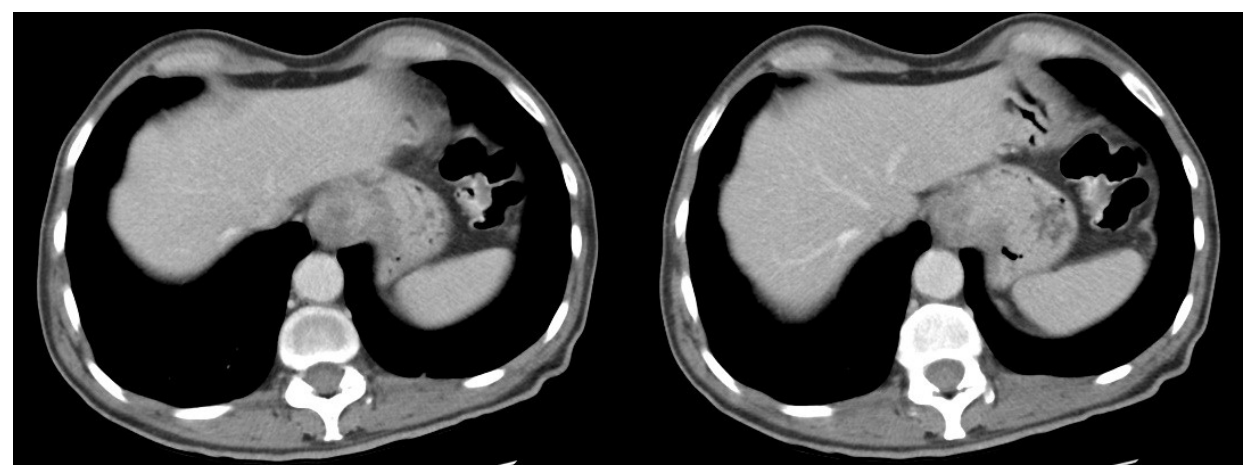

Figura 2: Tomografia computadorizada demonstrando espessamento parietal assimétrico na transição esofagogástrica.

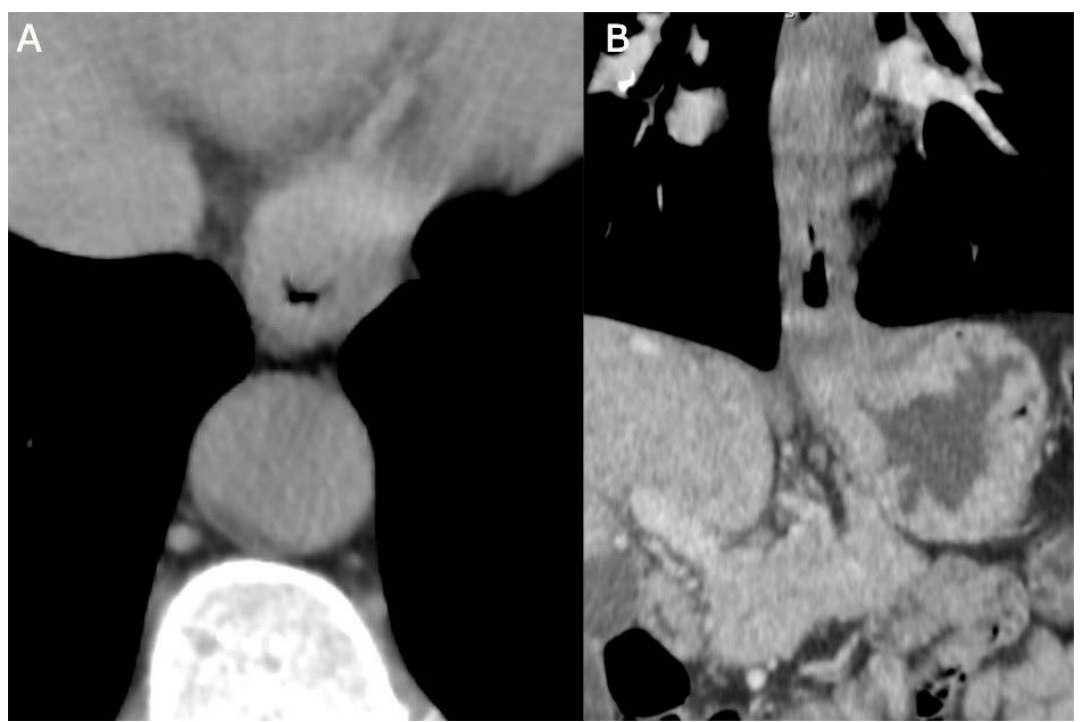

Figura 3: Tomografia computadorizada: A: Corte axial do esôfago distal com espessamento assimétrico; B: Reconstrução em coronal demonstrando espessamento que acomete a cárdia. 


\section{DISCUSSÃO}

Acalasia é uma doença motora primária de etiologia desconhecida em que ocorre falência no relaxamento do esfíncter esofágico inferior. A pseudoacalasia assemelha-se à acalasia na sua apresentação clínica, radiológica e endoscópica, sendo frequentemente muito difícil de distingui-la de acalasia na avaliação de rotina.

Idade avançada ( $\geq 55$ anos), curto tempo de sintomas ( $\leq 12$ meses), perda de peso significativa $(\geq 10 \mathrm{~kg}$ ) e dificuldade de passar endoscópio na junção esofagogástrica são fatores suspeitos para pseudoacalasia associada à malignidade. Considerando apenas os fatores de risco clínicos acima expostos, Ponds e colaboradores observaram que $88 \%$ dos pacientes com pseudoacalasia maligna possuíam 2 ou mais destes fatores comparado aos $25 \%$ encontrados no grupo de acalasia ${ }^{2}$.

Aspectos radiológicos auxiliares na diferenciação de acalasia de pseudoacalasia no exame contrastado são o comprimento do segmento estreitado do esôfago distal, o qual é frequentemente $<3,5 \mathrm{~cm}$ na acalasia enquanto na pseudoacalasia é $>3,5 \mathrm{~cm}$, e o grau de dilatação esofágica acima do segmento estreito, o qual é usualmente $>4 \mathrm{~cm}$ na acalasia devido ao longo tempo de obstrução enquanto que na pseudoacalasia é $<4 \mathrm{~cm}^{1}$.

Em casos de pseudoacalasia a tomografia computadorizada pode demonstrar espessamento assimétrico da parede esofágica (como no caso apresentado), massa ao redor da junção gastroesofágica, envolvimento linfonodal e disseminação regional e metastática ${ }^{1}$.

Autores utilizam a expressão síndrome de acalasia ao se referirem a sinais e sintomas comuns entre acalasia e pseudoacalasia ${ }^{3}$. Apesar dos aspectos clínicos, radiológicos e endoscópicos se assemelharem nas diferentes patologias, diferenciá-las têm implicações no tratamento e prognóstico dos pacientes.

\section{Conflitos de Interesse}

Os autores declaram que não tem conflito de interesse. Os dados utilizados neste relato estão de acordo com os preceitos éticos da instituição e foram aprovados pelo comitê de ética em pesquisa da ISCMPA.

\section{REFERÊNCIAS}

1. Abubakar U1, Bashir MB, Kesieme EB. Pseudoachalasia: A review. Niger J Clin Pract. 2016;19(3):303-7.
2. Ponds FA, van Raath MI, Mohamed SMM, Smout AJPM, Bredenoord AJ. Diagnostic features of malignancyassociated pseudoachalasia. Aliment Pharmacol Ther. 2017;45(11):1449-58.
3. Carlson DA, Kahrilas PJ. Editorial: when to be suspicious of malignancyassociated pseudoachalasia. Aliment Pharmacol Ther. 2017;46(2):198.

Recebido: 12 maio, 2020 Aceito: 18 nov, 2020 